\title{
Characterization of Clinical and Immune Response in a Rotavirus Diarrhea Model in Suckling Lewis Rats
}

\author{
FRANCISCO J. PÉREZ-CANO, MARGARIDA CASTELL, CRISTINA CASTELLOTE, AND ÀNGELS FRANCH
}

Department of Physiology, Faculty of Pharmacy, University of Barcelona, Spain E-08028 Barcelona

\begin{abstract}
Group A rotaviruses (RVs) are the leading pathogens causing diarrhea in children and animals. The present study was designed to establish an experimental model of RV infection and immune response in suckling rats. Wistar (W) and Lewis (L) suckling rats were inoculated orally with two different doses of a simian RV SA-11 strain. RV infection was evaluated by growth rate and clinical indexes. Virus-shedding and serum anti-RV antibodies were measured by enzyme-linked immunosorbent assay (ELISA). Mucosal interferon- $\gamma($ IFN $\gamma)$, specific splenocyte proliferation, and spleen and intestinal intraepithelial lymphocyte (IEL) phenotype were analyzed. No diarrhea was observed in any inoculated Ws. All Ls developed acute moderate diarrhea, and a high score and incidence of diarrhea were found in rats infected with higher titers of RV. Specific humoral and cell systemic immune response was confirmed by splenocyte proliferation and by the presence of serum anti-RV antibodies. Moreover, RV infection induced changes in IEL composition, which showed an increase in the proportion of innate immune cells with respect to cells involved in acquired immunity. This acute moderate diarrhea process constitutes a good experimental model that also provides some immune biomarkers that may allow establishing modulation by drugs or diet components. (Pediatr Res 62: 658-663, 2007)
\end{abstract}

$\mathrm{G}^{\mathrm{s}}$ roup A rotaviruses ( $\mathrm{RVs})$ - nonenveloped, icosahedral, and double-stranded RNA members of the family Reoviridae - are the leading pathogens causing diarrhea in children younger than $2 \mathrm{y}$. They infect enterocytes of the small intestine and cause severe gastroenteritis and dehydration; high morbidity is described worldwide, and symptomatic treatment such as rehydration is the only way to control this disease $(1,2)$. The pathophysiology of group A RV-associated diarrhea has been experimentally studied in several animal species and has helped to advance our knowledge about the infection process $(3,4)$. Recently, some experimental animal models have been very helpful in demonstrating that RV replication also exists outside the intestine, sera, and tissues through the detection of RNA or infectious viruses (5). RV infection in mice has been used to define some clinical features of the diarrhea process (4); however, only a few studies of RV infection have been performed in rats (3), and even fewer were focused on the immune response induced by the virus (6). The relationship of RV on the immune response

Received April 23, 2007; accepted June 28, 2007.

Correspondence: Francisco J. Pérez-Cano, Ph.D., Departament de Fisiologia, Facultat de Farmàcia, Av. Joan XXIII s/n, E-08028 Barcelona, Spain; e-mail: franciscoperez@ub.edu

This study was supported by grants from ISCIII (CB06/02/0079) and from the Generalitat de Catalunya SGCR-2005-00833. and in the development and resolution of disease has not been well established. Exceptionally, some works have focused on immune parameters, such as levels of specific antibody (Ab) in sera, feces, and intestine $(7,8)$.

Food supplementation with several compounds (i.e. probiotics, prebiotics, or protein concentrates) in RV-infected animals has been shown to provide a significant reduction in the incidence and severity of diarrhea as well as changes in laboratory parameters, such as viral shedding or RV concentration in intestinal segments $(8-10)$. Once again, enough immune biomarkers were not included in these studies.

In addition to the determination of disease parameters in $\mathrm{RV}$ infection, markers of immune response will provide clues about the mechanisms used for control, resolution, or prevention in subsequent infections. To better understand immune response against $\mathrm{RV}$ infections, this study was designed to develop a suckling rat RV infection model establishing some features of immune response, in addition to clinical indexes. Systemic immune response evaluation included lymphoproliferative assays and $\mathrm{Ab}$ production. Mucosal immune response evaluation incorporated intestinal IFN $\gamma$ production and changes in intraepithelial lymphocyte (IEL) composition. The characterization of this model may allow us to establish some immune biomarkers that could be used for the screening of any drug or dietary supplement proposed to treat or ameliorate diarrhea processes.

\section{MATERIALS AND METHODS}

Animals. Lewis (L) and Wistar (W) pregnant rats were obtained from Harlan (Barcelona, Spain). Pregnant rats were housed in individual cages under controlled temperature and humidity conditions in a $12 \mathrm{~h} / 12 \mathrm{~h}$ light/ dark cycle. Litters were unified to 10 pups per mother, with free access to the nipples and rat diet. Neonatal suckling rats (d 8 or 11 of life) were used for $\mathrm{RV}$ infection. Studies were performed in accordance with the institutional guidelines for the care and use of laboratory animals as established by the Ethical Committee for Animal Experimentation at the University of Barcelona and the Catalonia Government (CEEA-UB reference (Ref).74/05, DMA 3046).

Virus inoculation and experimental design. The simian SA-11 RV strain was purchased from the group Virus Entèrics of the University of Barcelona (A. Bosch and R. Pinto). The viruses were propagated in fetal African green monkey kidney (MA-104) cells and titered as plaque-forming units (PFUs). Rats were inoculated intragastrically with SA-11 virus at a low dose (LD) or high dose (HD), $10^{7}$ or $10^{8}$ PFUs per animal, respectively, in culture medium at $\mathrm{d} 8$ or 11 of life in each rat strain (10 animals per group). Inoculated animals

Abbreviations: Ab, antibody; DI, diarrhea index; DPI, day postinoculation; HD, high dose; IEL, intraepithelial lymphocyte; L, Lewis; $\mathbf{L D}$, low dose; NK, natural killer; PFU, plaque-forming unit; Ref, reference; RV, rotavirus; W, Wistar 
were maintained in separate isolators. Parallel reference (Ref) groups were established as a negative control for each strain and age (10 animals per group).

Clinical indexes and fecal specimen collection. SA-11 infection was evaluated from 1 to $14 \mathrm{~d}$ post-inoculation (DPI) by growth rate and clinical indexes such as severity of diarrhea, incidence of diarrhea (\%), and weight of fecal samples. Collection of individual fecal samples was performed once daily by gently pressing and massaging the abdomen, and samples were scored and weighed. Severity of diarrhea was assessed by fecal material examination and by scoring stools from 1 to 4 (diarrhea index [DI]) based on color, texture, and amount. Scoring was as follows: normal feces (score of 1), loose yellow-green feces (2), totally loose yellow-green feces (3), high amount of watery feces (4). Incidence of diarrhea corresponded to the percentage calculated by dividing the number of diarrheic samples by the number of total samples collected each day. Collected fecal samples were immediately frozen at $-20^{\circ} \mathrm{C}$ for further SA-11 particle quantification.

Intestinal samples collection. Rats were euthanized by humanitarian methods (one to two animals per DPI from each group) during the suckling period. Intestinal content was obtained by flushing in situ with $4 \mathrm{~mL}$ of cold saline solution. Intestinal tissue was cut into 5 - $\mathrm{mm}$ pieces and incubated with a phosphate-buffered solution (PBS) for $10 \mathrm{~min}$ at $37^{\circ} \mathrm{C}$ in a shaker. Supernatants from contents and washes were stored at $-80^{\circ} \mathrm{C}$ until IFN $\gamma$ quantification.

ELISA for SA-11 virus load in feces. Fecal samples were diluted in PBS $(20 \mathrm{mg} / \mathrm{mL})$ and homogenized by a Polytron (Kinematica, Switzerland). Homogenates were centrifuged, and supernatants were frozen at $-20^{\circ} \mathrm{C}$ until use. SA-11 particles in fecal samples were quantified by ELISA using polystyrene micro ELISA plates (Nunc Maxisorp, Wiesbaden, Germany), incubated with monoclonal Ab (MAb) anti-p42 (Biodesign Inc., Saco, ME). Remaining binding sites were blocked with PBS-1\% bovine serum albumin (BSA, $1 \mathrm{~h}$, room temperature [RT]). After washing, $100 \mu \mathrm{L}$ of appropriate diluted samples (fecal extract) in PBS-Tween-1\% BSA were added ( $3 \mathrm{~h}, \mathrm{RT})$. Both sheep anti-RV (group A) polyclonal Ab (Chemicon Inc., Temecula, CA) and donkey anti-sheep IgG biotin conjugate (Sigma Chemical Co., St. Louis, MO) were added, consecutively ( $1 \mathrm{~h}$ each). Then extravidin-peroxidase conjugate $(4 \mu \mathrm{g} / \mathrm{mL}$ in PBS-Tween-1\% BSA) was added for $30 \mathrm{~min}$ at RT. Captured SA-11 particles were detected by the addition of substrate solution (o-phenilenediamine plus hydrogen peroxide in $0.2 \mathrm{M}$ phosphate- $0.1 \mathrm{M}$ citrate buffer, $\mathrm{pH} 5$; Sigma Chemical Co.). Absorbance was measured at 492 $\mathrm{nm}$ after stopping the enzyme reaction with $3 \mathrm{M} \mathrm{H}_{2} \mathrm{SO}_{4}$. Dilutions of SA-11 particles were used as a standard in each plate.

ELISA for sera ANTI-SA-11 Ig quantification. Serum samples were obtained for anti-SA11 Ab quantification by ELISA. Ninety-six-well plates were incubated with SA-11 proteins $(10 \mu \mathrm{g} / \mathrm{mL})$ from sonicated virus suspension. After blocking with PBS-1\% BSA (1 h, RT), appropriate diluted sera in PBS-Tween-1\% BSA were added ( $3 \mathrm{~h}, \mathrm{RT})$. Abs were detected by adding rabbit anti-rat Ig conjugated to peroxidase (Sigma Chemical Co., $3 \mathrm{~h}$, RT) followed by enzyme substrate and stopping solutions as above. Pooled sera from RV inoculated animals were used as a standard in each plate.

ELISA for intestinal IFN $\gamma$ quantification. Levels of IFN $\gamma$ in intestinal content and washes were quantified using a rat IFN $\gamma$ OptEIA set from BD Pharmingen (Madrid, Spain).

Isolation and purification of splenocytes and IELs. At the end of the study period (14 DPI) rats inoculated with $10^{8}$ PFUs of SA-11 per animal on d 8 of life (L8-HD) and its Ref group (LRef) were sacrificed to obtain spleen mononuclear cells by mechanical disruption and density gradient purification, as previously described (11).

Intestinal IEL suspensions were isolated and purified from rats during the diarrhea process, on 1 and 3 DPI in L8-HD and LRef groups, as previously described (12). IELs were stained by Fluor chrome-conjugated MAb for flow cytometry analysis as previously described (11).

Specific stimulation and proliferation assays. Splenocytes from L8-HD and its Ref group on 14 DPI were incubated in quadruplicate in complete RPMI 1640 medium $\left(10^{5} / 200 \mu \mathrm{L}\right)$ with either sonicated SA-11 proteins (10 $\mu \mathrm{g} / \mathrm{mL}$ ) determined by the Bradford technique, inert protein (IP) at the same concentrations (BSA, $10 \mu \mathrm{g} / \mathrm{mL}$ ), or without stimulus in 96-well plates over $72 \mathrm{~h}$ at $37^{\circ} \mathrm{C}\left(5 \% \mathrm{CO}_{2}\right.$ in humidified air). Proliferating cells were identified by means of Biotrak cell proliferation system (Amersham Biosciences, Buckinghamshire, UK) according the manufacturer's instructions.

Statistical analysis. Results were expressed as mean \pm SEM. The software package SPSS 10.0 (SPSS Inc., Chicago, IL) was used for statistical analysis. Conventional one-way analysis of variance was performed, considering the experimental group as an independent variable. When virus inoculation had a significant effect on the dependent variable, the LSD test was applied. The Mann-Whitney $U$ test was used for nonparametric analysis. Significant differences were accepted when $p<0.05$.

\section{RESULTS}

Infection of $W$ suckling rats with SA-11. SA-11 infection was first evaluated in the $\mathrm{W}$ strain. Inoculation of $10^{8} \mathrm{PFUs}$ of SA-11 virus per animal on 8- or 11-d-old $\mathrm{W}$ suckling rats (W8-HD and W11-HD, respectively) did not modify their body weight increase with respect to noninoculated W (WRef) (Fig. 1A). No animals from the W11-HD group developed diarrhea (Fig. $1 B$ ), and only $20 \%$ of W8-HD-infected animals developed a mild diarrhea at 2 DPI, with severity scores of only around 2. After these clinical results, this strain was rejected as a rat model for immune RV response evaluation, and no other determinations were done.

Infection of L suckling rats with SA-11 and clinical evaluation. After discarding the $\mathrm{W}$ strain, $\mathrm{L}$ were inoculated with SA-11 with two different doses $\left(10^{7}\right.$ PFUs per animal [LD] or $10^{8}$ PFUs per animal (HD), to two different groups, based on animal age (8- or 11-d-old rats). RV inoculation did not modify body weight increase in any L experimental group. All the groups showed a parallel growth with regard to the reference group (Fig. 2A).

All L inoculated groups (L8-HD, L8-LD, and L11-HD) developed diarrhea as was observed by incidence results (Fig. $2 B$ ) and the DI (Fig. 2C). Most of L8-LD and L11-HD groups developed mild diarrhea (DI 2) or no diarrhea (DI $<2$ ) (Fig. $2 C$ ) during 1-6 DPI, and the proportion of animals that developed disease only exceeded $50 \%$ on 4 and $2-3$ DPI, respectively (Fig. 2B). On the other hand, a high incidence (up to $90 \%$ ) of RV infection of L8-HD rats with diarrhea was found at 2 and 4 DPI (Fig. 2B). These animals developed a biphasic disease with two peaks of infection on 2 and 4 DPI.
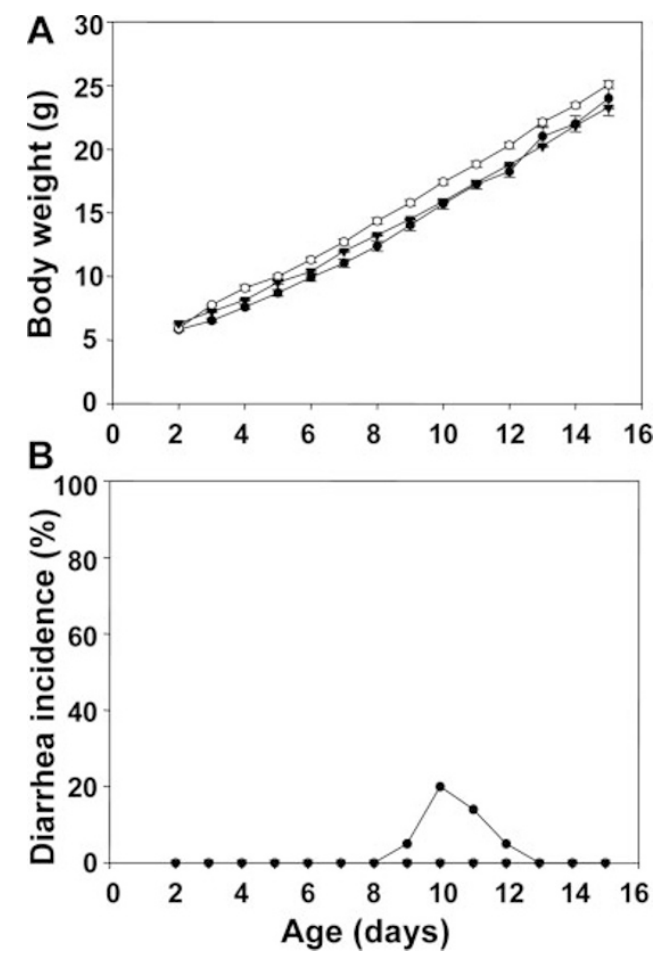

Figure 1. Body weight $(A)$ and diarrhea incidence $(\%)(B)$ throughout the study period in W suckling rats inoculated with RV SA-11 and Ref groups: W8-HD (๑), W11-HD (O), and WRef $(\boldsymbol{\Lambda})$. Values are expressed as mean \pm SEM $(n=10)$. 

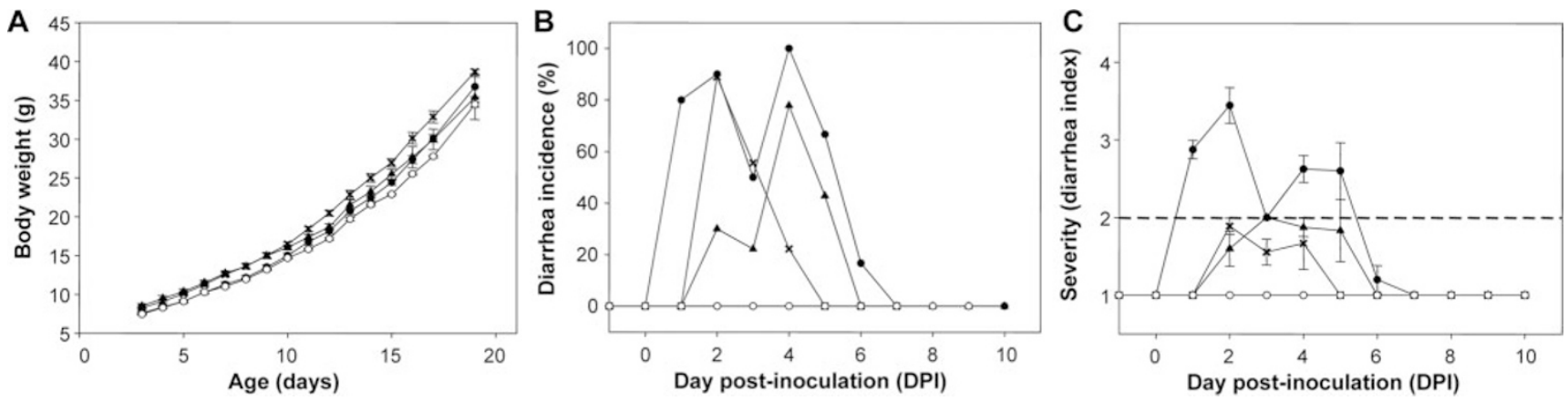

Figure 2. Body weight $(A)$, diarrhea incidence $(\%)(B)$, and severity of diarrhea (DI) $(C)$ throughout the study period in L suckling rats inoculated with RV SA-11 and Ref groups: L8-LD $(\mathbf{\Lambda})$, L8-HD $(\bullet)$, L11-HD ( $(\boldsymbol{\bullet})$, and LRef $(\bigcirc)$. Individual data correspond to the mean \pm SEM $(n=6-10)$.

Table 1. Clinical indexes of the time course of diarrhea

\begin{tabular}{lccc}
\hline & \multicolumn{1}{c}{ L8-LD } & L8-HD & \multicolumn{1}{c}{ L11-HD } \\
\hline MDI & $2 \pm 0.0(9)$ & $3.5 \pm 0.2(9)^{* \dagger}$ & $2 \pm 0.0(9)$ \\
BDD & $3.1 \pm 0.3(9)$ & $1.2 \pm 0.1(10)^{* \dagger}$ & $2.1 \pm 0.1(9) \ddagger$ \\
EDD & $5.1 \pm 1.0(7)$ & $5.8 \pm 0.3(6)^{* \dagger}$ & $3.8 . \pm 0.8(9) \ddagger$ \\
DP & $1.9 \pm 0.3(7)$ & $4.7 \pm 0.3(9)^{* \dagger}$ & $1.7 \pm 0.3(9)$ \\
Maximum incidence, \% & 80 & 90 & 90
\end{tabular}

Some parameters describing severity of disease (MDI), timing and length of the diarrhea process (BDD, EDD, and DP [calculated by difference from the EDD with respect to the BDD]) for each animal are shown. Maximum incidence of diarrhea was achieved at different time points during the DP. Each datum corresponds to the mean \pm SEM $(n)$.

Statistical differences: * $p<0.05$ L8-HD $v s$ L8-LD; $\uparrow p<0.05$ L8-HD $v s$ L11-HD; $\ddagger p<0.05$ L11-HD vs L8-LD.

To better compare groups, some related clinical severity indexes were established (Table 1). Animals from L8-HD showed significantly higher maximum DI (MDI) than the other two inoculated groups $(p<0.05)$. Moreover, in L8-HD group, diarrhea started earlier (established by beginning diarrhea day $[\mathrm{BDD}]$ ) and finished later (established by ending diarrhea day [EDD]) than both other infected groups $(p<$ 0.05 ). Thus, the duration of the total diarrhea process (established by diarrhea period [DP]) was also approximately 2.5 times longer than the others $(p<0.05)$.

Fecal weight and SA-11 viral shedding. Significant fecal weight increase was found in both inoculated groups (Fig. $3 A$ ), L8-LD and L8-HD, during the DP between 1 and 4 DPI, when it is compared with LRef animals $(p<0.05)$. The fecal weight gain was only observed in DP because later, when clinical disappeared (7-12 DPI) fecal weight was similar to that from LRef animals.

Viral load was analyzed in L8-HD animals during the postinfection period with maximum virus shedding immediately after inoculation. In addition, the bimodal pattern found in incidence and severity of diarrhea appeared here again on 1-2 and 5 DPI (Fig. 3B), whereas the load of fecal RV particles was almost undetectable after 6 DPI by ELISA.

Mucosal immune response. In the L8-HD group, mucosal cell immune response was evaluated. Phenotype of effector cells in intraepithelial (IE) compartment was analyzed by establishing main subsets: T CD8 + IEL (IE-T), intraepithelial natural killer (NK) cells (IE-NK), and IE-NKT cells. RV infection induced changes in IEL composition, which were
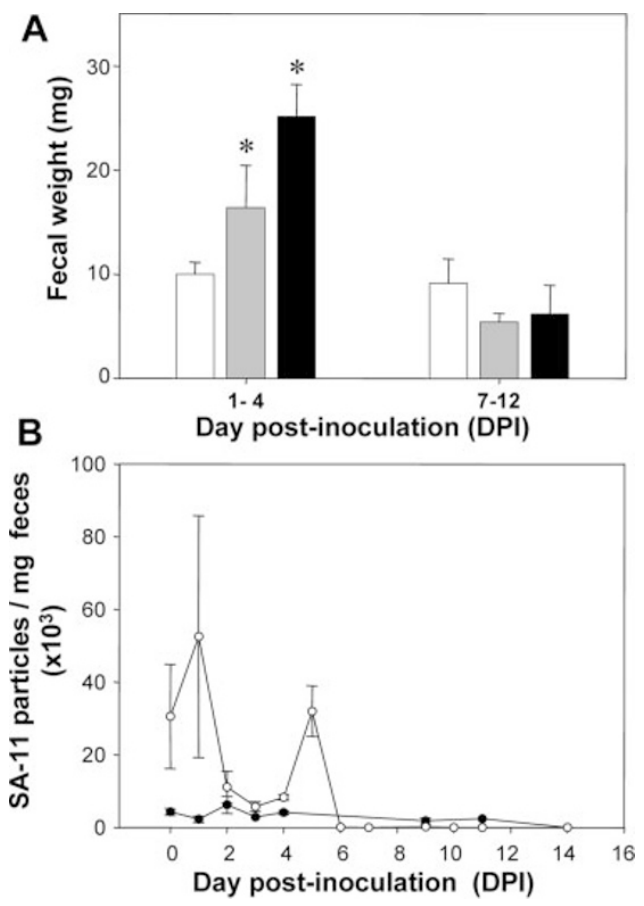

Figure 3. (A) Fecal weight during the DP (between 1 and 4 DPI) and when clinical signs disappeared (7-12 DPI) in L suckling rats inoculated on 8-d-old animals with RV SA-11 and Ref groups: L8-LD (shaded columns), L8-HD (solid columns), and LRef (open columns). (B) Viral antigen-shedding curves of individual fecal samples of L rats inoculated with RV SA-11 and Ref groups from inoculation day to $14 \mathrm{DPI}$ : L8-HD $(\bigcirc)$ and LRef $(\bullet)$. Results are expressed as mean $\pm \operatorname{SEM}(n=6-10) . * p<0.05$ between SA-11-infected animals and its corresponding Ref group (LRef).

already present on 1 DPI (data not shown) and more evident at 3 DPI (Fig. 4A). Thus, at 3 DPI, inoculation induced a lower proportion of IE-T. On the other hand, a significant increase in IE-NK cell proportion and a significant decrease in IE-NKT cell proportion was observed in L8-HD group, in relation to reference animals (27\% and 29\%, respectively). As CD8 $\alpha \alpha+$ IEL includes both CD8 + IE-NK cells and some IE-T, and CD $8 \alpha \beta+$ IEL includes IE-NKT cells and some IE-T, the $\mathrm{CD} 8 \alpha \alpha / \mathrm{CD} 8 \alpha \beta$ ratio in total gated IELs may represent the ratio between innate immune cells with respect to cells involved in more acquired immune processes in the intestine. In this sense, $\mathrm{CD} 8 \alpha \alpha / \mathrm{CD} 8 \alpha \beta$ ratio in L8-HD rats at 3 DPI increased approximately three times with respect to LRef animals at the same age (11 d) (data not shown, $p<0.05$ ). 
A

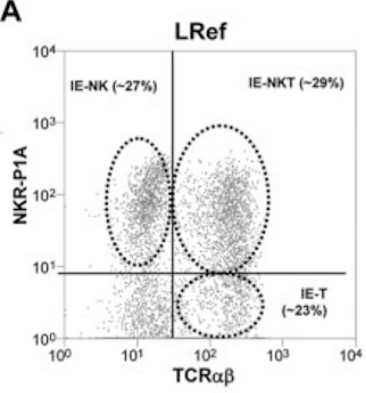

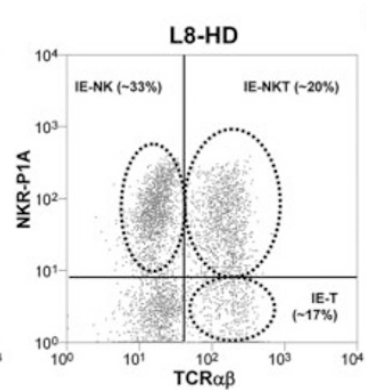
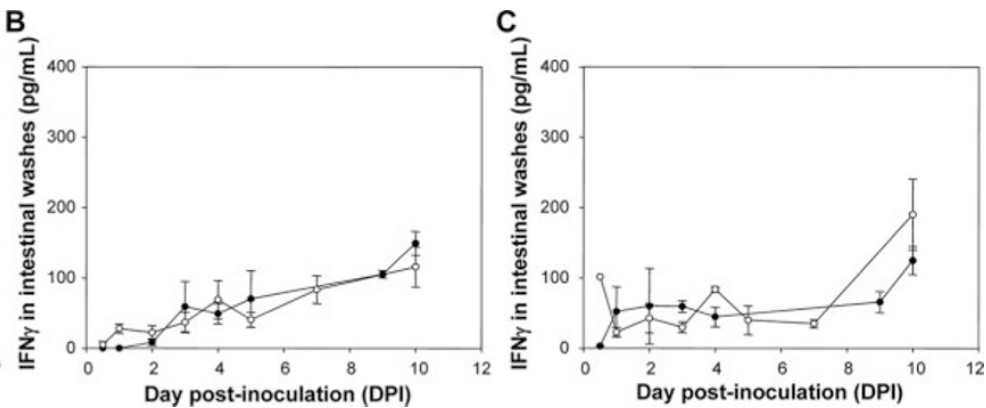

Figure 4. (A) IEL phenotype at 3 DPI in L rats inoculated on 8-d-old animals with RV SA-11 and Ref groups: L8-HD (O) and LRef (-). Representative cytograms at 3 DPI of the distribution frequency of fluorescence intensity obtained by TCR $\alpha \beta /$ NKR-P1A MAb labeling of IELs. Marked regions include three cell subsets: TCR $\alpha \beta-$ NKR-P1A + cells (IE-NK), TCR $\alpha \beta+$ NKR-P1A + cells (IE-NKT), and TCR $\alpha \beta+$ NKR-P1A- cells (IE-T). The percentage of each subset (shown in the figure) corresponds to the mean value derived from three to five animals. Time course of IFN $\gamma$ levels quantified in intestinal washes $(B)$ and in intestinal content $(C)$, determined by ELISA, during the postinfection period in L suckling rats inoculated with RV SA-11 (L8-HD) and Ref (LRef) groups. Results are expressed as mean $\pm \operatorname{SEM}(n=2-6)$.

Mucosal IFN $\gamma$ production remained very low at an early age $(<100 \mathrm{pg} / \mathrm{mL})$ in both inoculated and Ref animals as shown in Figure $4 B$ and $C$. IFN $\gamma$ levels in intestinal content were low from inoculation day (8-d-old animals) to 9 DPI, but later those levels tended to increase. IFN $\gamma$ in intestinal washes showed an age-dependent behavior $(p<0.01)$ from the first day (8-d-old animals) to the last of the period studied (18 d old), when values increased five to seven times. Despite these observed patterns, IFN $\gamma$ levels were so low in intestinal content and washes that significant differences between infected and noninoculated animals could not be established.

Systemic humoral and cell immune response. Specific humoral immune response was evaluated by quantification of serum anti-RV Ab in inoculated animals despite the early age of the animals; all RV-inoculated rats produced high titers of a specific $\mathrm{Ab}$ against $\mathrm{RV}(2.3 \pm 0.09$ Absorbance Units, mean \pm SEM, $n=6$ ), determined by ELISA at the end of the study period, thus developing good immune response against SA-11 infection.

On the other hand, systemic cell immune response was confirmed by specific splenocyte proliferation against SA-11 particles on 14 DPI (Fig. 5). Spleen cells from L8-HD group showed high ability to specifically proliferate under SA-11

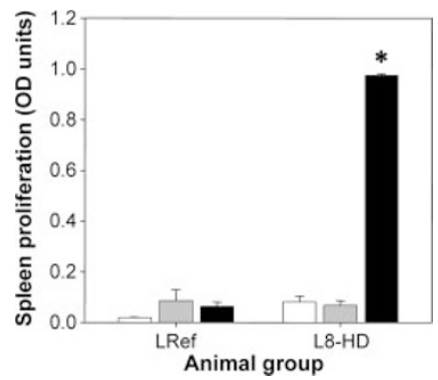

Figure 5. Specific splenocyte proliferative response. Splenocytes were obtained from $\mathrm{L}$ suckling rat litters inoculated on 8-d-old animals with RV SA-11 (L8-HD) and Ref (LRef) groups at 14 DPI and cultured with $10 \mu \mathrm{g} / \mathrm{mL}$ of SA-11 proteins (solid columns), inert protein (IP) at same concentration (shaded columns), or without stimulus (NS, open columns). Results are showed as Absorbance Units obtained in the same plate and expressed as the mean \pm SEM of three to five values, each one derived from quadruplicates of one suckling animal. $* p<0.05$ between SA-11-infected animals and its corresponding reference group (LRef). stimulation conditions with respect to LRef splenocytes or other culture conditions (i.e. inert protein added to the plate) $(p<0.05)$.

\section{DISCUSSION}

Very few studies provide experimental data of group A RV infections in rats, an animal species that is widely used to develop experimental models for pathophysiological studies. Some of these studies have been developed in germ-free suckling rats (13), but Ciarlet et al (3) developed an extensive work in the characterization of diarrhea process in suckling $\mathrm{L}$, with some heterologous RV strains. We set up our studies in $\mathrm{W}$ and $\mathrm{L}$ strains, and in older suckling animals $(8$ and $11 \mathrm{~d}$ old). As the diarrhea process is induced later, the more mature have the immune response developed to control the infection; moreover, longer preventive or ameliorating approaches can be tested.

In this study, $\mathrm{W}$ rats were first considered, despite the scarce literature regarding the susceptibility of this rat strain to $\mathrm{RV}$ infection. It has been suggested that some rat strains seem to be resistant to $\mathrm{RV}$ infection due to alterations in the natural entry of the virus into the intestinal epithelial cells, which is based on a complex multistep process (14-16). Due to the lack of precise information about RV infection in $\mathrm{W}$ rats, $\mathrm{RV}$-associated diarrhea in $\mathrm{W}$ rats was performed by high-titer inoculation $\left(10^{8}\right.$ PFUs per animal) of the SA-11 strain. However, only a couple of animals inoculated on d 8 of life developed mild diarrhea. Our results demonstrated that $\mathrm{W}$ rats do not develop group A RV diarrhea, may be lacking the protein that mediates RV-cell attachment or inhibition of RV replication by certain mucins, as previously suggested $(17,18)$.

Although, all 8- and 11-d-old L rats infected groups with SA-11 developed diarrhea without affecting their growth pattern, the mean incidence and severity diarrhea were higher in 8-d-old animals inoculated with high titer of SA-11 strain $\left(10^{8}\right.$ PFUs per animal). As expected, age and dose markedly influenced the severity of the disease, with the earliest inoculation (at $8 \mathrm{~d}$ of life) and the highest dose (HD) registering the most potent effect on diarrhea process established by clinical indexes. The significant increase in fecal sample weight col- 
lected during the DP found here was also described in other studies, as is the case of RV-induced germ-free rats (13). Thus, the features determined in our rat suckling model of 8-d-old L rats infected with $10^{8} \mathrm{RVs}$ per animal (L8-HD), which develop an acute and moderate diarrhea process, provide a good experimental model of acute infectious diarrhea in conventional rats.

Furthermore, clinical studies and results from animal models showed the ability of RV to spread to the bloodstream and infect peripheral tissues besides the intestine (19). Thus, RV can be detected in a broad array of organs, and its presence may explain some clinical consequences exhibited by infected children $(20,21)$. Otherwise, protection mechanisms and interaction with the immune system have been less studied, and thus evaluation of cell/humoral and mucosal/systemic immune responses have become the focus of attention in the present work.

It should be noted that rat suckling immune responses are limited and less competent than those developed by adult animals. In this sense, in previous studies, we have shown age-related changes in lymphocyte composition and functionality in neonatal rat spleen and mucosal lymphocytes (11,12,22).

Immune response against $\mathrm{RV}$ infection must take place first at mucosal sites. For this reason, mucosal IFN $\gamma$ production and IEL phenotype were analyzed in small intestine. IFN $\gamma$ production at intestinal sites, as a mediator of effector T-cell response, was analyzed in intestinal washes and content in 8-d-old animals inoculated with high titer of SA-11 strain (L8-HD). No differences between inoculated and Ref groups were found, in agreement with other studies in mice infected with RV (23). In this sense, these results suggest that neonatal immune response, regarding IFN $\gamma$ production involved in $\mathrm{RV}$ clearance, seems to be limited and less competent than that in adult age. Otherwise, it is possible that IFN $\gamma$ does not have a pivotal role in diarrhea resolution because IFN $\gamma$ knockout mice are able to control this type of infection (24).

Because an important proportion of $\mathrm{RV}$-specific $\mathrm{T}$ and innate immune cells may remain in an IE compartment to control the infection, main effector IEL subsets were investigated. In previous studies, we demonstrated that in early rat life, the intestinal compartment includes a high proportion of NK cells $(12,21)$, which should have an important role in the defense during this stage of development. Current studies also suggest the predominance of IE-NKT during suckling (unpublished observations). We describe here, for the first time, changes in some populations induced by $\mathrm{RV}$ infection. Changes in IE-NK, IE-NKT, and IE-T cell subset proportion demonstrate that innate immune cell percentages are clearly increased after RV infection, based on the rise of the CD8 $\alpha \alpha$ / $\mathrm{CD} 8 \alpha \beta$ ratio in total gated IEL. The increase in this ratio reflects the rise in IE-NK and IE-T CD $8 \alpha \alpha+$ cells-innate immunity - and the respective decrease in IE-T CD $8 \alpha \beta+$ cells - acquired immunity or IE-NKT cells - bridge between innate and acquired immunity (25). This fact suggests that innate immune response is the arm of the immune system that is in charge of controlling this kind of viral infection at mucosal sites at an early age, when acquired immunity is still not fully developed.

Because an important proportion of $\mathrm{RV}$-specific $\mathrm{T}$ and innate immune cells may remain in the IE compartment to control the infection, main effector IEL subsets were investigated. We describe here, for the first time, changes in these populations induced by $\mathrm{RV}$ infection. Changes in IE-NK, IE-NKT, and IE-T cell subset proportion demonstrate that innate immune cell percentages are clearly increased after RV infection, based on the rise of $\mathrm{CD} 8 \alpha \alpha / \mathrm{CD} 8 \alpha \beta$ ratio in total gated IEL. This fact suggests that innate immune response is the arm of the immune system that is in charge of controlling this kind of viral infection in mucosal sites at an early age, when acquired immunity is still not fully developed.

It has been described that serum $\operatorname{IgA}$ titers correlate with protection from RV disease and high titers correlate with protection from reinfection (26). As suckling rodents still produce low levels of mucosal IgA, the main source of this Ig (21), we analyzed serum-specific anti-SA-11 total Ig in inoculated animals, and our results confirmed that these animals presented high titers of anti-RV Ig, as other authors found in stools (8).

As spleen lymphocytes of inoculated rats are capable of proliferating in response to antigen in vitro, we suggest that either primed lymphocytes have migrated to extraintestinal tissues such as the spleen or viral particles have arrived at other tissues where cells have been stimulated. Nevertheless, lymphoproliferative ability may be used as a good biomarker of specific immune responses against RV infection. The assay was performed after the active infection process at $14 \mathrm{DPI}$; thus, it may reflect the presence of memory lymphocytes and so may represent a marker of defense against reinfection.

In summary, this study provides a self-limiting acute RVinduced diarrhea experimental model that does not affect pup development and contributes to the understanding of the mechanisms involved in immune protection and resolution of the diarrhea process and prevention of reinfection. More importantly, it provides some immune response biomarkers such as lymphocyte phenotype and proliferative ability, which may help to evaluate the activity of several food compounds not only by shortening the diarrhea process, but also enhancing the specific RV immune response.

Acknowledgments. The authors thank X. Abad for his work on SA-11 production and S. Marín-Gallén for her help with laboratory work.

\section{REFERENCES}

1. Leung AK, Kellner JD, Davies HD 2005 Rotavirus gastroenteritis. Adv Ther 22:476-487

2. Vuletic B, Obradovic S, Stojkovic-Andjelkovic A, Igrutinovic Z, Radlovic P 2006 [Rotavirus gastroenteritis]. Srp Arh Celok Lek 134:166-169

3. Ciarlet M, Conner ME, Finegold MJ, Estes MK 2002 Group A rotavirus infection and age-dependent diarrheal disease in rats: a new animal model to study the pathophysiology of rotavirus infection. J Virol 76:41-57

4. Blutt SE, Fenaux M, Warfield KL, Greenberg HB, Conner ME 2006 Active viremia in rotavirus-infected mice. J Virol 80:6702-6705

5. Blutt SE, Conner ME 2007 Rotavirus: to the gut and beyond. Curr Opin Gastroenterol 23:39-43

6. Jaimes MC, Rojas OL, Gonzalez AM, Cajiao I, Charpilienne A, Pothier P, Kohli E, Greenberg HB, Franco MA, Angel J 2002 Frequencies of virus specific CD4(+) and 
CD8(+) $\mathrm{T}$ lymphocytes secreting gamma interferon after acute natural rotavirus infection in children and adults. J Virol 76:4741-4749

7. Eydelloth RS, Vonderfecht SL, Sheridan JF, Enders LD, Yolken RH 1984 Kinetics of viral replication and local systemic immune responses in experimental rotavirus infection. J Virol 50:947-950

8. Qiao H, Duffy LC, Griffiths E, Dryja D, Leavens A, Rossman J, Rich G, RiepenhoffTalty M, Locniskar M 2002 Immune responses in rhesus rotavirus-challenged BALB/c mice treated with bifidobacteria and prebiotic supplements. Pediatr Res 51:750-755

9. Guerin-Danan C, Meslin JC, Chambard A, Charpilienne A, Relano P, Bouley C, Cohen J, Andriex C 2001 Food supplementation with milk fermented by Lactobacillus casei $\mathrm{DN}-114001$ protects suckling rats from rotavirus/associated diarrhea. J Nutr 131:111-117

10. Wolber FM, Broomfield AM, Fray L, Cross ML, Dey D 2005 Supplemental dietary whey protein concentrate reduces rotavirus-induced disease symptoms in suckling mice. J Nutr 135:1470-1474

11. Pérez-Cano FJ, González-Castro AM, Marín-Gallén S, Castellote C, Franch A, Castell M Phenotypic and functional characteristics of rat spleen lymphocytes during suckling. Dev Comp Immunol (in press)

12. Pérez-Cano FJ, Castellote C, González-Castro A, Pelegrí C, Castell M, Franch A 2005 Developmental changes in intraepithelial T lymphocytes and NK cells in the small intestine of neonatal rats. Pediatr Res 58:885-891

13. Guerin-Danan C, Meslin J, Lambre F, Charpilienne A, Serezat M, Bouley C, Cohen J, Andrieux C 1998 Development of a heterologous model in germfree suckling rats for studies of rotavirus diarrhea. J Virol 72:9298-9302

14. Arias CF, Isa P, Guerrero CA, Néndez E, Zárate S, Lopez T, Espinosa Ó, Romero P, Lopez S 2002 Molecular biology of rotavirus cell entry. Arch Med Res 33:356-361

15. Isa P, Arias CF, Lopez S 2006 Role of sialic acids in rotavirus infection. Glycoconj J 23:27-37
16. Lopez S, Arias CF 2006 Early steps in rotavirus cell entry. Curr Top Microbiol Immunol 309:39-66

17. Bass DM, Mackow ER, Greenberg HB 1991 Identification and partial characterization of a rhesus rotavirus binding glycoprotein on murine enterocytes. Virology 183:602-610

18. Yolken RH, Ojeh C, Khatri IA, Sajjan U, Forstner JF 1994 Intestinal mucins inhibit rotavirus replication in an oligosaccharide-dependent manner. J Infect Dis 169:1002-1006

19. Ramig RF 2004 Pathogenesis of intestinal and systemic rotavirus infection. J Virol 78:10213-10220

20. Crawford SE, Patel DG, Cheng E, Berkova Z, Hyser JM, Ciarlet M, Finegold MJ, Conner ME, Estes MK 2006 Rotavirus viremia and extraintestinal viral infection in the neonatal rat model. J Virol 80:4820-4832

21. Fenaux M, Cuadras M, Feng N, Jaimes M, Greenberg H 2006 Extraintestinal spread and replication of a homologous EC rotavirus strain and a heterologous Rhesus rotavirus in BALB/c mice. J Virol 80:5219-5232

22. Pérez-Cano FJ, Castellote C, Marín-Gallén S, Franch A, Castell M 2005 Neonatal immunoglobulin secretion and lymphocyte phenotype in rat small intestine lamina propria. Pediatr Res 58:164-169

23. Fromantin C, Piroth L, Peitpas I, Pothier P, Kohli E 1998 Oral delivery of homologous and heterologous strains of rotavirus to BALB/c mice induces the same profile of cytokine production by spleen cells. Virology 244:252-260

24. Franco MA, Greenberg HB 1997 Immunity to rotavirus in T cell deficient mice. Virology 238:169-179

25. Taniguchi M, Harada M, Kojo S, Nakayama T, Wakao H 2003 The NKT cell system: bridging innate and acquired immunity. Annu Rev Immunol 21:483513

26. Matson DO 1996 Protective immunity against group A rotavirus infection and illness in infants. Arch Virol Suppl 12:129-139 